\title{
Practice patterns regarding drains management in breast surgery: Results of a survey of Senonetwork Italia breast centers
}

\section{Tiziano Pallara MD ${ }^{1}$ (D) | Lucio Fortunato $M^{1}$ | Secondo Folli $M^{2}$ | \\ Manuela Roncella MD ${ }^{3}$ | Nicolò Scuderi MD, $\mathrm{PhD}^{4}$ | Daniele Friedman MD, $\mathrm{PhD}^{5}$ | \\ Zoran Arnez MD, PhD ${ }^{6}$ | Diego Ribuffo MD, PhD ${ }^{4}$ | Elena Manna MD $^{1}$ | \\ Paolo Persichetti MD, PhD ${ }^{7}$ Senonetwork Italia Breast Centres Responders}

\footnotetext{
${ }^{1}$ Azienda Ospedaliera San Giovanni-Addolorata, Roma, Italy

${ }^{2}$ Fondazione IRCCS Istituto Nazionale dei Tumori di Milano, Milano, Italy

${ }^{3}$ Azienda Ospedaliero Universitaria Pisana, Pisa, Italy

${ }^{4}$ Università degli Studi di Roma "La Sapienza", Roma, Italy Trieste, Italy

${ }^{7}$ Università Campus Bio-Medico di Roma, Roma, Italy

Correspondence

Tiziano Pallara, A.O. San Giovanni Addolorata, Via dell'Amba Aradam 9, 00184 Rome (RM), Italy. Email: t.pallara@gmail.com
}

${ }^{5}$ IRCCS Azienda Ospedaliera Universitaria San Martino - IST - Istituto Nazionale per la Ricerca sul Cancro, Genova, Italy

${ }^{6}$ Dipartimento delle Scienze Mediche, Chirurgiche e della Salute, ASUITS Azienda Sanitaria Universitaria Integrata di Trieste, Università degli Studi di Trieste,

Surgery for breast cancer has changed in the last few decades in favor of more conservative approaches, without compromising locoregional control and survival. Common immediate complications following breast surgery are hematoma, seroma, and wound infection. ${ }^{1}$ Traditionally, surgeons have implemented the use of closed-suction drains in this setting with the aim of preventing these complications, which can cause discomfort, morbidity, increased follow-up visits, and possible delay in the beginning of adjuvant therapies. ${ }^{2}$ On the other hand, potential benefits of performing breast procedures without using a drain have been increasingly considered. ${ }^{3}$ There is lack of modern and official guidelines on use of drains in breast surgery, with no consensus regarding their management and the appropriate use of antibiotics in this setting. Almost all reports agree with the use of drains after breast procedures, especially if they are associated with reconstruction or axillar lymph node dissection (ALND), but an extreme variability is reported regarding practice patterns. ${ }^{4}$

A national survey regarding the management of drains after breast cancer surgery was sent by email on January 2018 to all Breast Centers (BC) registered with Senonetwork Italia. Data collected by the administrative office of Senonetwork were de-identified and exported for statistical analysis. A Steering Committee

Senonetwork Italia Breast Centres Responders are present in Appendix 1. composed of general surgeons and plastic surgeons was appointed to coordinate the survey, which consisted of 22 multiple choice questions, designed to inquire about surgeons' demographics, use of drains after breast procedures with and without reconstruction and/or ALND, antibiotic use and to characterize drain management in their clinical practice.

A total of $105 \mathrm{BC}$, performing more than 40000 breast cancer cases each year, were surveyed electronically and 73 complete responses were returned (72\%).

All 73 evaluable respondents described themselves as breast surgeons, among whom 48 (63\%) performed both breast cancer surgery and reconstruction. Most respondents $(n=34 ; 45 \%)$ reported that more than 300 cases of breast cancer operations were performed in their BC each year.

In cases of breast conservative surgery, 18 (25\%) BC affirmed that they use "always" a drain, 19 (26\%) "never," and 36 (49\%) "sometimes." The choice of the latest answer was often associated with the increased volume of the breast, the quantity of breast parenchyma asportation, the need of glandular flap for oncoplastic purpose, and the presence of high-risk factors for hematoma and/or seroma. 
In cases of implant-based breast reconstruction, 51 (70\%) BCs used two drains, one placed in the submuscular pocket and one in the subcutaneous space. When only one drain was used (30\%), it was placed subcutaneously or in the submuscular pocket in $16 \%$ and in $14 \%$ of cases, respectively.

If ALND was associated with a mastectomy, one, two, or three drains were used in $3 \%, 60 \%$, and $37 \%$, respectively.

The gauge of drains used was $14-16 \mathrm{Fr}$ in $56 \%$ of cases, $<14 \mathrm{Fr}$ in $15 \%$, while more than $16 \mathrm{Fr}$ in $29 \%$ of cases.

Respondents stated that hospitalization was not prolonged for the presence of drains and that discharge of the patients occurred 24 hours after the operation in 50\% of cases, while they affirmed that drains "sometimes" affect hospitalization by two to three days, or "always" prolonged it more than three days in $43 \%$ and $7 \%$ of cases, respectively.

A dedicated nurse service was involved in the drain management in 57 (78\%) cases, while in 16 (22\%) this was not present.

Shoulder immobilization after ALND and/or breast reconstruction was not adopted by 56 (77\%) BC, while shoulder immobilization was "always" or "sometimes" applied in 12 (16\%) and 5 (7\%) cases, respectively.

Drains were removed when 24 hours output was $<50 \mathrm{cc},<30 \mathrm{cc}$, after 24 hours, and after $4-7$ days in 55\%, 34\%, 3\%, and $8 \%$ of cases, respectively.

Sixty-six (90\%) respondents reported adopting a routine practice of using antibiotics beyond 24 hours, while 7 (10\%) BC did not use postoperative antibiotic prophylaxis.

Among respondents who reported postoperative prophylactic antibiotic use, the duration of treatment was variable. Antibiotic prophylaxis for "2-5 days" or "until all drains were removed" was reported in $53 \%$ and $22 \%$ of cases, respectively, especially if breast reconstruction was performed.

Many differences were found among postoperative antibiotics. First-generation cephalosporins, second- or third-generation cephalosporins, amoxicillin plus clavulanic acid, and fluoroquinolones were used in $25 \%, 25 \%, 34 \%$, and $7 \%$ of cases, respectively.

When asked to comment about the clinical relevance of drains both in the present and in the future, $88 \%$ of respondents reported that, nowadays, drains are of clinical relevance in breast surgery, and $63 \%$ stated that they will continue to be important in the next future.

We report the first national survey in Italy regarding the use of drains in breast cancer surgery.

Data showed that the use of drains was associated with a prolonged hospitalization in $50 \%$ of BC. This finding is consistent with an analysis of five systematic reviews which concluded that the insertion of a drain is associated with a longer hospital stay and reduced seroma formation. ${ }^{5}$

Half of BC surveyed removed drains when the 24 hours output was $<50 \mathrm{cc}$. To date, the correct "timing" of the removal of drains remains unclear and literature reports do not provide precise indications. Some studies, especially in the setting of breast reconstruction, show evidence supporting late removal $(<30 \mathrm{~mL}$ drainage in
24 hours or postoperative day 14$)^{6}$, while others stress the advantages of early removal (postoperative day 2 , regardless of volume). ${ }^{7}$

Antibiotic use has always been an important topic in breast surgery. In a survey of the American Society of Breast Surgeons $(\mathrm{ASBrS})$, cephalosporins were utilized uniformly as preoperative antibiotic prophylaxis. ${ }^{8}$ This is consistent with the Surgical Care Improvement Project guidelines established in 2005 and confirmed in $2015 .{ }^{9}$ In our survey, this recommendation was adopted by only $25 \%$ of BC.

Use of postoperative antibiotic prophylaxis has been strongly associated with the presence of immediate breast reconstruction after mastectomy. Despite a Cochrane study, a meta-analysis and recent guideline's recommendations stated that for clean and clean-contaminated procedures additional prophylactic antimicrobial agents should not be administered after the surgical incision is closed in the operating room, even in the presence of a drain, ${ }^{10}$ our survey confirmed this lack of uniformity regarding both the timing of antibiotic discontinuation and the type of antibiotic eventually used.

In this national survey, we report a large variability regarding drain use in the breast conservative setting. After mastectomy or axillary node dissection, drains are usually employed and generally removed when 24 hours output was $<50 \mathrm{cc}$. Consistent with literature data, their use has still an impact on the hospital length of stay. Surgeons generally continue antibiotic prophylaxis during the 24 perioperative hours and antibiotic use is still controversial regarding both the timing of discontinuation and the type used, despite precise guidelines present in literature. Furthermore, according to our results, most surveyed surgeons believe drains play a clinically relevant role in breast surgery and that they will continue to have importance in the future.

\section{ORCID}

Tiziano Pallara (iD https://orcid.org/0000-0001-7138-8098

\section{REFERENCES}

1. Edwards JR, Peterson KD, Mu Y, et al. National Healthcare Safety Network (NHSN) report: data summary for 2006 through 2008, issued December 2009. Am J Infect Control. 2009;37:783-805.

2. Kuroi K, Shimozuma K, Taguchi T, et al. Evidence-based risk factors for seroma formation in breast surgery. Jpn J Clin Oncol. 2006;36(4):197-206.

3. Baker E, Piper J. Drainless mastectomy: is it safe and effective? Surgeon. 2017;15(5):267-271.

4. Brett T, Wang ED, Mirrer J, et al. Current practice among plastic surgeons of antibiotic prophylaxis and closed-suction drains in breast reconstruction. Ann Plast Surg. 2011;66:460-465.

5. Ebner F, deGregorio N, Vorwerk E, Janni W, Wöckel A, Varga D. Should a drain be placed in early breast cancer surgery? Breast Care. 2014;9:116-122.

6. Barton A, Blitz M, Callahan D, Yakimets W, Adams D, Dabbs K. Early removal of postmastectomy drains is not beneficial: results from a halted randomized controlled trial. Am J Surg. 2006;191(5):652-656. 
7. Chapman D, Purushotham AD. Acceptability of early discharge with drain in situ after breast surgery. Br J Nurs. 2002;10(22):1447-1450.

8. Brahmbhatt RD, Huebner M, Scow JS, et al. National practice patterns in preoperative and postoperative antibiotic prophylaxis in breast procedures requiring drains: survey of the American Society of Breast Surgeons. Ann Surg Oncol. 2012;19(10):3205-3211.

9. The Joint Commission. Surgical care improvement project (SCIP). http://www.jointcommission.org/surgical_care_improvement project/. Accessed November 3, 2015.

10. Berríos-Torres SI, Umscheid CA, Bratzler DW, et al. Centers for disease control and prevention guideline for the prevention of surgical site infection, 2017. JAMA Surg. 2017;152(8):784-791.

\section{APPENDIX 1}

\section{SENONETWORK ITALIA BREAST CENTRES RESPONDERS}

Sergio Abonante, Cosenza-Azienda Ospedaliera Cosenza; Vittorio Altomare, Roma-Policlinico Universitario Campus Bio-Medico; Claudio Amanti, Roma-A.O. Sant'Andrea Sapienza Università di Roma; Luciana Ambrosiani, Como-Ospedale Valduce; Claudio Andreoli, Castellanza-Humanitas Mater Domini (VA); Alberto Bafile, L'Aquila-Ospedale San Salvatore; Bettina Ballardini, Milano-Multimedica S.P.A.; Maggiorino Barbero, Asti-Ospedale Cardinal Massaia; Claudio Battaglia, Sanremo-Ospedale Civile Di Sanremo (IM); Anna Bianchi, Brescia-Spedali Civili Di Brescia; Laura Biganzoli, Prato-ASL Toscana Centro, Ospedale S Stefano Prato; Marina Bortul, Trieste-Dipartimento Scienze Mediche Chirurgiche e della Salute (DSMCS) Trieste-ASUITS, Ospedale di Cattinara; Patrizia Bravetti, Ravenna-Centro Prevenzione Oncologica; Stefano Burlizzi, Brindisi-Ospedale A. Perrino; Carlo Cabula, Cagliari-C.R.O. di Riferimento "A. Businco"; Claudio Caponi, Empoli-Ospedale S Giuseppe ASL Toscana Centro (FI); Francesco Caruso, Catania-Humanitas Centro Catanese Di Oncologia; Carla Cedolini, Udine-Azienda Sanitaria Universitaria Integrata; Ettore Cianchetti, Chieti Ortona-G. Bernabeo; Fabio Corsi, Pavia-Istituti Clinici Scientifici Maugeri IRCCS - Milano, Dipartimento di Scienze; Biomediche e Cliniche L. Sacco, Università degli Studi di Milano; Annalisa Curcio, Forlì-Ausl Forlì; Olindo Custodero, Bari-Breast Unit Ospedale San Paolo Asl Bari; Francesco D'Errico, MonzaPoliclinico Di Monza; Secondo Folli, Milano-Fondazione IRCCS Istituto Nazionale dei Tumori di Milano; Lucio Fortunato, RomaAzienda Ospedaliera San Giovanni-Addolorata; Duilio Francesconi, Lido Di Camaiore-Ospedale Versilia UsI Toscana Nord Ovest (LU); Antonio Frassoldati, Ferrara-Cona Arcispedale S. Anna; Patrizia Frittelli, Roma-Fatebenefratelli Isola Tiberina; Daniele Generali,
Cremona-Azienda Socio-Sanitaria Territoriale di Cremona; Riccardo Giovanazzi, Monza-Ospedale San Gerardo; Massimo Maria Grassi, Bergamo-Humanitas Gavazzeni; Alessandra Huscher, BresciaFondazione Poliambulanza; Maria Grazia Lazzaretti, Carpi-Ospedale Ramazzini (MO); Francesco Leone, Catanzaro-Azienda Ospedaliera Pugliese-Ciaccio; Gianfranco Lolli, Foligno-San Giovanni Degli Infermi - Foligno (PG); Carla Magni, Lecco-SSD "Breast Unit" ASST Lecco; Pietro Mainente, Vicenza -Schio-Ospedale Alto VicentinoCentro Donna; Luigi Manca, Lecce-Città Di Lecce Hospital; Stefano Mancini, Milano-A.O. Polo Universitario Luigi Sacco; Samuele Massarut, Aviano-Centro di Riferimento Oncologico di Aviano (PN); Alberto Massocco, Negrar-IRCCS Sacro Cuore Don Calabria, Negrar, Verona (VR); Lorenzo Menghini, Santarcangelo Di RomagnaOspedale degli Infermi di Rimini (RN); Maria Mirri, Roma-Asl Roma 1 - Ospedale S. Spirito - S. Filippo Neri; Guido Mondini, Ivrea-Breast Unit Ovidio Paino Aslto4 (TO); Massimo Monti, Roma-Policlinico Umberto Primo; Roberto Murgo, San Giovanni Rotondo-IRCCS Casa Sollievo Della Sofferenza (FG); Maria Grazia Pacquola, Tortona-S.O.S. Senologia Tortona Asl Alessandria (AL); Adriana Paduos, Biella-Ponderano-Asl Biella; Gianmatteo Pagani, MilanoDivisione di Senologia Chirurgica - Istituto Europeo di Oncologia; Claudio Pagliari, Desenzano-Azienda Ospedaliera Desenzano del Garda (VR); Dante Palli, Piacenza-Ospedale Guglielmo Da Saliceto; Guido Papaccio, Venezia-Ospedale All'angelo Mestre e SS Giovanni E Paolo; Matteo Passamonti, Lodi-Azienda Socio Sanitaria Territoriale (ASST) di Lodi; Alba Pellegrini, Faenza-Presidio Faenza Ausl Romagna (RA); Francesca Pellini, Verona-Azienda Ospedaliera Universitaria Integrata Verona; Francesca Pietribiasi, MoncalieriAsl TO 5 Ospedale Santa Croce (TO); Cosmo Maurizio Ressa, Bari-IRCCS Istituto Tumori Giovanni Paolo II Bari; Fabio Ricci, Latina-Ospedale S. M. Goretti; Manuela Roncella, Pisa - Azienda Ospedaliero Universitaria Pisana; Francesca Rovera, VareseAsst-Settelaghi Ospedale di Circolo Varese; Corrado Rubino, Salerno-A.O.U. San Giovanni di Dio e Ruggi D'Aragona; Enzo Maria Ruggeri, Viterbo-Ospedale Belcolle Ausl Viterbo; Antonio Rulli, Perugia-Azienda Ospedaliero-Universitaria Di Perugia; Alessandro Sanguinetti, Terni-Azienda Ospedaliera Terni; Maurizio Saturno, Potenza-Azienda Ospedaliera Regionale San Carlo; Giuliano Scalco, Vicenza-Ospedale Civile San Bortolo; Adele Sgarella, PaviaFondazione IRCCS Policlinico San Matteo Pavia; Paolo Stefanini, Cinisello Balsamo-Istituti Clinici di Perfezionamento Bassini Sesto (MI); Francesca Svegliati, Roma-A.O.S.Camillo-Forlanini; Mario Taffurelli, Bologna-Policlinico di Sant'Orsola - Bologna; Giovanni Tazzioli, Modena-Policlinico Di Modena; Corrado Tinterri, Rozzano-Humanitas Cancer Center (MI); Martino Trunfio, NapoliOspedale Cardarelli; Pasquale Zagarese, Benevento-A.O San Pio Benevento. 